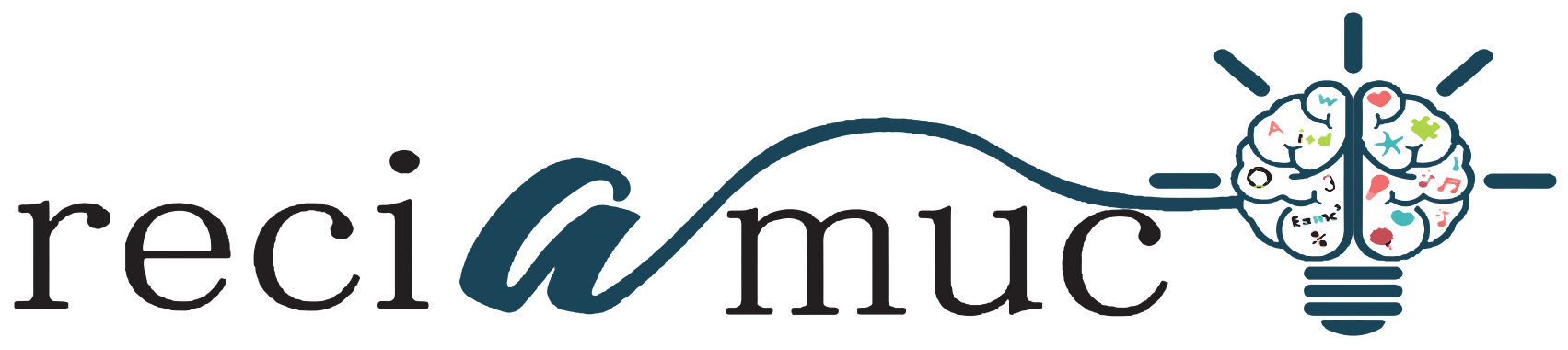

DOI: 10.26820/reciamuc/5.(3).agosto.2021.132-139

URL: https://reciamuc.com/index.php/RECIAMUC/article/view/704

EDITORIAL: Saberes del Conocimiento

REVISTA: RECIAMUC

ISSN: 2588-0748

TIPO DE INVESTIGACIÓN: Artículo de Revisión

CóDIGO UNESCO: 32 Ciencias Médicas

PAGINAS: $132-139$

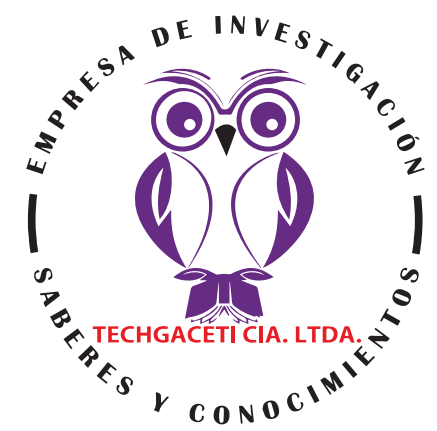

\title{
Síndrome inflamatorio multisistémico en el adulto
}

Multisystemic inflammatory syndrome in the adult Síndrome inflamatória multissistêmica no adulto

Melissa Fernanda Mera Cáceres'; Valeria Tatiana Arroyo Valẹncia2; Cristian Fabricio Rojas Carvajal3; Carolina Misshell Narváez Álvarez ${ }^{4}$

RECIBIDO: 10/06/2021 ACEPTADO: 12/07/2021 PUBLICADO: 31/08/2021

1. Médico Residente Asistencial en hospital Fuerzas Armadas; Quito, Ecuador; melissameracaceres@gmail.com; (D) https://orcid.org/0000-0003-3969-3521

2. Médico General; Investigador Independiente; Quito, Ecuador; tatianarroyo1608@hotmail.com; (D) https://orcid. org/0000-0002-0784-5538

3. Médico General; Investigador Independiente; Quito, Ecuador; rojascristiank94@gmail.com; (D) https://orcid. org/0000-0001-5647-2013

4. Médica Cirujana; Investigador Independiente; Quito, Ecuador; caro93narvaez@gmail.com; (D) https://orcid. org/0000-0003-2354-2497

CORRESPONDENCIA

Melissa Fernanda Mera Cáceres

melissameracaceres@gmail.com

Quito, Ecuador

๑) RECIAMUC; Editorial Saberes del Conocimiento, 2021 


\section{RESUMEN}

Desde junio de 2020, se han publicado varios informes de casos y series que informan de un síndrome inflamatorio multisistémico en adultos, como respuesta inflamatoria severa retardada a la infección por SARS-CoV-2 y que Ileva a daño en múltiples órganos, como el corazón, los vasos sanguíneos, los riñones, el sistema digestivo, el cerebro, la piel o los ojos. Entre los síntomas compatibles con esta patología, destacan: fiebre que se prolonga por 24 horas o más y latidos cardíacos irregulares; evidencia de disfunción cardíaca; síntomas gastrointestinales y erupciones cutáneas. El tratamiento para esta patología depende del tipo y de la gravedad de los síntomas y de qué órganos y otras partes del cuerpo se ven afectados por la inflamación, y generalmente se basa en inmunomoduladores, tales como corticosteroides y/o plasmaféresis, dado que se han reportado resistencias a la inmunoglobulina intravenosa. El objetivo de este trabajo investigativo, desarrollado bajo una metodología documental, se centra en realizar una revisión a la literatura científica disponible, que principalmente aborde al Síndrome inflamatorio multisistémico en el adulto, cómo detectar esta patología, cuales son los síntomas y los diversos tratamientos a aplicar, así como recomendar medidas de prevención, todo ello con la finalidad de proporcionar un material actualizado que defina dichos aspectos. Para ello se escogieron más de 12 fuentes digitales, ubicadas en portales tales como: Centro para el Control y la Prevención de Enfermedades, Clínica Mayo, intramed, entre otras, que fundamentan las ideas aquí planteadas, y que consecuentemente sirven de base para concluir que se trata de una enfermedad compleja cuyo abordaje en los adultos aún está en investigación, por tratarse de una patología que inicialmente había sido presentada solo en pacientes pediátricos, y que teniendo en cuenta que está relacionado con la enfermedad COVID-19, es muy importante cumplir con las medidas de prevención recomendadas para la infección por SARS-CoV-2.

Palabras clave: Síndrome inflamatorio, multisistémico, SARS-COV-2, infección, inflamación.

\section{ABSTRACT}

Since June 2020, several case reports and series have been published reporting a multisystemic inflammatory syndrome in adults, as a delayed severe inflammatory response to SARS-CoV-2 infection and leading to damage to multiple organs, such as the heart. , blood vessels, kidneys, digestive system, brain, skin, or eyes. Among the symptoms compatible with this pathology, the following stand out: fever that lasts for 24 hours or more and irregular heartbeat; evidence of cardiac dysfunction; gastrointestinal symptoms and skin rashes. Treatment for this condition depends on the type and severity of symptoms and on which organs and other parts of the body are affected by inflammation, and is generally based on immunomodulators, such as corticosteroids and / or plasmapheresis, since they have been reported resistance to intravenous immunoglobulin. The objective of this investigative work, developed under a documentary methodology, is focused on conducting a review of the available scientific literature, which mainly addresses the multisystemic inflammatory syndrome in adults, how to detect this pathology, what are the symptoms and the various treatments to apply, as well as recommend prevention measures, all with the aim of providing updated material that defines these aspects. For this, more than 12 digital sources were chosen, located in portals such as: Center for Disease Control and Prevention, Mayo Clinic, intramed, among others, which support the ideas raised here, and that consequently serve as a basis to conclude that It is a complex disease whose approach in adults is still under investigation, as it is a pathology that had been presented only in pediatric patients, and taking into account that it is related to the COVID-19 disease, it is very important to comply with recommended prevention measures for SARS-CoV-2 infection.

Keywords: Inflammatory syndrome, multisystem, SARS-COV-2, infection, inflammation.

\section{RESUMO}

Desde junho de 2020, vários relatos de casos e séries foram publicados relatando uma síndrome inflamatória multissistêmica em adultos, como uma resposta inflamatória grave retardada à infecção por SARS-CoV-2 e levando a danos a múltiplos órgãos, como o coração. , vasos sanguíneos, rins, sistema digestivo, cérebro, pele ou olhos. Dentre os sintomas compatíveis com essa patologia, destacam-se: febre de 24 horas ou mais e batimento cardíaco irregular; evidência de disfunção cardíaca; sintomas gastrointestinais e erupções cutâneas. O tratamento para essa condição depende do tipo e da gravidade dos sintomas e de quais órgãos e outras partes do corpo são afetados pela inflamação, e geralmente é baseado em imunomoduladores, como corticosteroides e / ou plasmaférese, uma vez que foi relatada resistência à injeção intravenosa imunoglobulina. O objetivo deste trabalho investigativo, desenvolvido sob uma metodologia documental, está centrado em realizar uma revisão da literatura científica disponível, que aborda principalmente a síndrome inflamatória multissistêmica em adultos, como detectar esta patologia, quais os sintomas e os diversos tratamentos para aplicar, bem como recomendar medidas de prevenção, tudo com o objetivo de disponibilizar material atualizado que defina esses aspectos. Para isso, foram escolhidas mais de 12 fontes digitais, localizadas em portais como: Centro de Controle e Prevenção de Doenças, Clínica Mayo, intramed, entre outras, que sustentam as ideias aqui levantadas e que consequentemente servem de base para a conclusão de que é uma doença complexa cuja abordagem em adultos ainda está em investigação, por se tratar de uma patologia que se apresentou apenas em pacientes pediátricos, e por se tratar de doença COVID-19, é muito importante cumprir as recomendações medidas de prevenção da infecção por SARS-CoV-2.

Palavras-chave: Síndrome inflamatória, multissistema, SARS-COV-2, infecção, inflamação. 


\section{Introducción}

El síndrome inflamatorio multisistémico en niños (MIS-C) es una complicación rara pero grave de la infección por SARS-CoV-2 en niños y adolescentes. Desde junio de 2020, se han publicado varios informes de casos y series que informan de un síndrome inflamatorio multisistémico similar en adultos (MIS-A). (Bamrah, y otros, 2020).

El síndrome inflamatorio multisistémico en adultos (SIM-A) "es un trastorno infrecuente; se caracteriza por un espectro heterogéneo de manifestaciones clínicas y a menudo pasa desapercibido. Se requieren estudios prospectivos para mejorar el abordaje diagnóstico y terapéutico de los pacientes con esta entidad." Davogustto et al. (2021)

No existe ninguna prueba que permita confirmar o descartar la presencia de esta enfermedad. Sin embargo, se pueden utilizar criterios como los descritos por Storch \& Vinagre (2020):

Para diagnosticarla se utilizan unos criterios clínicos, entre los que se incluyen la fiebre persistente y la afectación de varios órganos o sistemas (cardíaco, respiratorio, hematológico, gastrointestinal, dermatológico o neurológico). Para ayudar al diagnóstico, se solicitará un análisis de sangre buscando signos de inflamación. Además, tras el diagnóstico se deberá realizar una ecografía cardíaca para descartar la afectación del corazón.

Esta patología causa síntomas provocados por la inflamación (irritación, dolor e hinchazón) de todo el cuerpo. Entre los síntomas más comunes, se incluyen los señalados por (Thacker, 2021):

- Fiebre de varios días de duración.

- Dolor abdominal.

- Vómitos o diarrea.

- Erupción en la piel.

- Labios rojos, secos y agrietados.
- Ojos rojos.

- Hinchazón de manos o pies.

- Dolor en las articulaciones.

- Mareos.

- Problemas en la vista.

- Dolor de cabeza.

- Palidez.

El reconocimiento del síndrome inflamatorio multisistémico tanto en adultos como en niños fortalece la necesidad de esfuerzos de prevención para limitar la propagación del SARS-CoV-2, entre los que Storch \& Vinagre (2020) sugieren:

- Lavado frecuente de manos con agua y jabón o soluciones hidroalcohólicas.

- Uso de mascarilla en todos los lugares públicos.

- Mantener la distancia de seguridad de 1,5 metros con personas no convivientes.

- Evitar lugares cerrados o con aglomeración de personas.

- Al toser o estornudar, taparse la nariz y la boca con el codo o un pañuelo desechable.

- Evitar tocarse los ojos, la nariz y la boca.

- Ante cualquier síntoma sospechoso, quedarse en el domicilio y avisar a su médico.

El objetivo de este trabajo investigativo, desarrollado bajo un metodología documental, se centra en realizar una revisión a la literatura científica disponible, que principalmente aborde al Síndrome inflamatorio multisistémico en el adulto, cómo detectar esta patología, cuales son los síntomas y los diversos tratamientos a aplicar, así como recomendar medidas de prevención, todo ello con la finalidad de proporcionar un material actualizado que defina dichos aspectos y 
que sea de utilidad no solo para el personal de salud, sino también para el público en general, por lo que para ello se estructura de la siguiente manera: definiciones, medios diagnóstico, sintomatología, tratamiento y medidas de prevención.

\section{Materiales y Métodos}

El presente trabajo de investigación, enmarcado en una metodología documental, se centra en realizar una revisión a la literatura científica disponible, con el objeto de construir un material bibliográfico actualizado, enfocado en dar a conocer los criterios vigentes del Síndrome inflamatorio multisistemico en el adulto.

Para ello se escogieron más de 12 fuentes digitales, ubicadas en portales tales como: La Organización Mundial de la Salud (OMS), Centro para el Control y la Prevención de Enfermedades, Clínica Mayo, Intramed, entre otras especializadas en el área de salud. Como términos de búsqueda se utilizaron las expresiones "Síndrome inflamatorio", "Síndrome inflamatorio multisistémico", "Síndrome inflamatorio y SARS-Cov-2". Y se aplicaron criterios de selección tales como: idioma español; publicación entre 2020 y 2021 (ambos inclusive), acceso completo y abierto; en el área de salud y medicina; estudios referidos a humanos; tipo de bibliografía, artículos científicos, manuales médicos, guías clínicas, ensayos clínicos, estudios o reportes de casos, noticias científicas, boletines y/o folletos de instituciones oficiales o privadas de excelente trayectoria en el área de la salud, medicina o científico académica, y demás, monografías y otros documentos que, a criterio propio, mostraran información de interés en base a la observación de la evidencia científica referida en sus contenidos. Este proceso arrojó resultados que en promedio oscilaron entre 7 y 69 enlaces a fuentes de información bibliográficas.

De tal manera fueron adelantadas otras búsquedas menores sin considerar la aplicación de cualquier otro criterio de descar- te, ya que se requirió encontrar información complementaria que independientemente de su origen o época de publicación, es considerada igualmente importante ajustada y de relevancia para este tema.

Es a partir de entonces que se procedió con la lectura crítica y análisis interpretativo de un pilar de información recopilado a lo largo de la investigación, que también fue adoptada como evidencia. Resultando todo este proceso en la selección de los elementos más sustanciales y significativos de las diferentes fuentes bibliográficas que fundamentan el razonamiento aquí expuesto.

\section{Resultados}

Desde junio de 2020, se han publicado varios informes de casos y series que informan de un síndrome inflamatorio multisistémico en adultos (MIS-A, siglas en inglés), según Rivera (2020):

El síndrome inflamatorio multisistémico, (MIS-C), es una complicación rara pero grave de la infección por el coronavirus, que hasta hace poco solo se presentaba en niños y adolescentes, y ahora se está viendo en adultos. Los casos notificados a los Centros de Control y Prevención de Enfermedades (CDC) y las series e informes de los casos publicados identifican síndrome inflamatorio multisistémico en adultos (MIS-A), que generalmente requiere cuidados intensivos y puede tener resultados fatales, ya que la enfermedad inflama varios órganos vitales.

El síndrome inflamatorio multisistémico en adultos (SIM-A), "es una afección grave que puede inflamar algunos órganos del cuerpo, como el corazón, los vasos sanguíneos, los riñones, el sistema digestivo, el cerebro, la piel o los ojos. La inflamación suele incluir hinchazón, enrojecimiento y dolor." (Theimer , 2020).

Esta patología presenta un cuadro clínico que evidencia una "respuesta inflamatoria severa retardada a la infección por SARS- 
CoV-2 y que lleva a daño en múltiples órganos, ya sean pacientes previamente sanos o con comorbilidades, y que cursan con infección reciente o actual por COVID-19, o que tienen un nexo epidemiológico claro." Giraldo et al. (2020).

\section{Diagnóstico}

El síndrome multisistémico inflamatorio, se presenta en adultos que previamente se infectaron con el virus que causa la COVID-19 y muchos no supieron que lo tenían. Como lo indica artículo publicado por la Clínica Mayo (2021):

MIS-A parece presentarse semanas después de la infección con la COVID-19, aunque algunos adultos están infectados al mismo tiempo. Si se sospecha la presencia del MIS-A, una prueba diagnóstica o de anticuerpos para la COVID-19 puede ayudar a confirmar una infección presente o pasada con el virus, lo que ayuda con el diagnóstico para el MIS-A.

Además de la prueba de anticuerpos Heredia (2021) establece los siguientes criterios clínicos para el diagnóstico:

1. Enfermedad severa que requiere hospitalización en una persona de 21 años o más.

2. Resultado positivo, en una prueba, de una infección actual o pasada por SARSCoV-2, durante la admisión o dentro de las 12 semanas previas (ácido nucleico, antígeno, anticuerpos).

3. Disfunción severa de 1 o más sistemas de órganos extrapulmonares (por ejemplo, hipotensión arterial o shock, trombosis venosa o arterial, tromboembolismo, falla hepática aguda).

4. Evidencia de laboratorio de inflamación (proteína C reactiva, ferritina, dimero D, interleuquina-6 elevadas).

5. Ausencia de enfermedad respiratoria severa (para excluir que la disfunción de órganos es debido a hipoxia tisular).
La demora temporal en el diagnóstico del síndrome inflamatorio multisistemico, puede generar graves complicaciones en quienes padecen esta patología, por lo que "es de suma importancia la sospecha y reconocimiento temprano de la entidad, como la rápida oferta de tratamiento médico agresivo y en las unidades de complejidad adecuadas (cuidados intermedios/intensivos) con el fin de prevenir el desarrollo de secuelas como los aneurismas de arterias coronarias, entre otros." Brizuela et al. (2021).

\section{Sintomatología}

Los adultos que se han recuperado del Covid-19 pueden desarrollar síndrome inflamatorio multisistémico, y "los médicos deben considerar esta posibilidad en adultos con síntomas específicos, como disnea leve, fatiga profunda, anorexia y fiebre de hasta $40^{\circ} \mathrm{C}$, conjuntivitis, cambios en la mucosa oral, así como un agrandamiento de los ganglios linfáticos del cuello." (García, 2021).

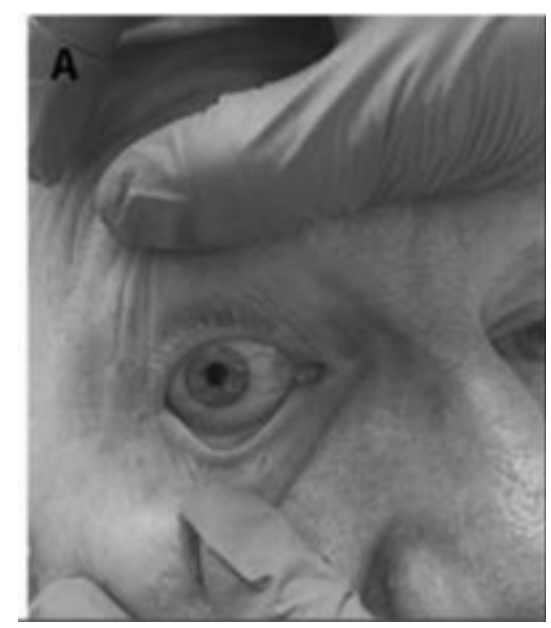

Figura 1. Conjuntivitis Bilateral.

Recuperado de: El síndrome inflamatorio multisistémico postCovid es posible en adultos. García (2021). https://www.redaccionmedica.com/secciones/sanidad-hoy/ el-sindrome-inflamatorio-multisistemico-postcovid-es-posible-en-adultos-1638 
El Centro para el Control y la Prevención de Enfermedades (CDC) (2020) señala los siguientes síntomas en pacientes con SIM-A:

- Fiebre.

- Dolor estomacal.

- Ojos inyectados de sangre.

- Diarrea.

- Mareos o sensación de desmayo (signos de presión arterial baja).

- Sarpullido.

- Vómitos.

- Dificultad para respirar.

- Dolor o presión persistente en el pecho.

- Confusión.

- Incapacidad de despertarse o permanecer despierto.

- Piel, labios o lechos de uñas pálidos, grises o azulados, según el tono de la piel.

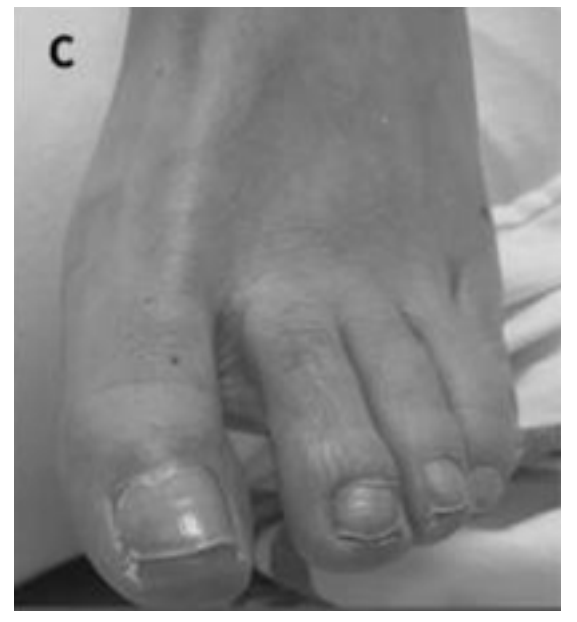

Figura 2. Dedos del pie con eritema maculopapular.

Recuperado de: El síndrome inflamatorio multisistémico postCovid es posible en adultos. García (2021). https://www.redaccionmedica.com/secciones/sanidad-hoy/ el-sindrome-inflamatorio-multisistemico-postcovid-es-posible-en-adultos-1638
Entre otros de los síntomas compatibles con esta patología, destacan: "fiebre que se prolonga por 24 horas o más y latidos cardíacos irregulares; evidencia de disfunción cardíaca; síntomas gastrointestinales y erupciones cutáneas. Las radiografías pueden mostrar inflamación pulmonar incluso si los pacientes no presentan síntomas." (Fox, 2020).

\section{Tratamiento}

A los pacientes adultos con Síndrome inflamatorio multisistémico se les han administrado diversas terapias, que según Mendoza et al. (2021) incluyen:

Tratamiento con inmunoglobulina intravenosa, corticosteroides, y el inhibidor del receptor de IL-6 tocilizumab. La mayoría requiere cuidados intensivos. Los mecanismos patológicos específicos que impulsan MIS-A siguen siendo desconocidos, y se necesitan más datos sobre los factores de riesgo, las características clínicas y los resultados para guiar las intervenciones terapéuticas.

El tratamiento para esta patología depende del tipo y de la gravedad de los síntomas y de qué órganos y otras partes del cuerpo se ven afectados por la inflamación, y generalmente "se basa en inmunomoduladores tales como corticosteroides y/o plasmaféresis, dado que se han reportado resistencias a la inmunoglobulina intravenosa. Sin embargo, la inmunoglobulina intravenosa parece ser eficaz en pacientes adultos." Elouardi et al. (2020)

\section{Prevención}

No se puede prevenir el desarrollo de esta forma de la enfermedad. Sin embargo, teniendo en cuenta que está relacionado con la enfermedad COVID-19, es muy importante cumplir con las medidas higiénicas recomendadas para la prevención de la infección por SARS-CoV-2, señaladas por la Organización Mundial de la Salud (OMS) (2021): 
- Siga las orientaciones locales: Tome conocimiento de las orientaciones de las autoridades nacionales, regionales y locales, a fin de contar con la información más pertinente al lugar en que se encuentre.

- Guarde la distancia adecuada: Manténgase al menos a un metro de otras personas, incluso aunque no parezcan estar enfermas, puesto que es posible tener el virus sin manifestar síntomas.

- Póngase mascarilla: Utilice una mascarilla de tres capas que se ajuste bien, especialmente cuando no sea posible mantener la distancia física, o en interiores.

- Evite los lugares concurridos, los interiores mal ventilados y el contacto prolongado con otras personas. Pase más tiempo al aire libre que en interiores.

- La ventilación es importante: Abra las ventanas de los lugares cerrados con el fin de aumentar el volumen de aire exterior.

- Evite tocar superficies, especialmente en entornos públicos o en los establecimientos de salud, por si las ha tocado alguna persona con COVID-19. Limpie periódicamente las superficies con desinfectantes normales.

- Lévese frecuentemente las manos con agua y jabón o con un gel hidroalcohólico. De ser posible, lleve consigo gel hidroalcohólico y utilícelo con frecuencia.

- Al toser o estornudar, cúbrase la boca y la nariz con el codo flexionado o con un pañuelo y tire inmediatamente los pañuelos usados en una papelera cerrada. A continuación, lávese las manos o desinféctelas con gel hidroalcohólico.

- Vacunarse: Cuando le llegue el turno, vacúnese. Seguir las orientaciones y recomendaciones locales sobre la vacunación.

\section{Conclusión}

Se necesitan más investigaciones para comprender las causas y el desarrollo de esta enfermedad así como sus efectos a largo plazo. El reconocimiento del síndrome inflamatorio multisistemico tanto en adultos como en niños fortalece la necesidad de esfuerzos de prevención para limitar la propagación del SARS-CoV-2.

Es indispensable la concientización de los profesionales de salud respecto esta patología, su diagnóstico temprano y tratamiento apropiado con el fin de disminuir las complicaciones que podrían incluso tener desenlaces fatales.

\section{Bibliografía}

Bamrah, S., Schwartz, N., Patel, P., Abbo, L., Beauchamps, L., Balan, S., . . . Geevarughese, A. (03 de 10 de 2020). Intramed. Recuperado el 04 de 08 de 2021, de https://www.intramed.net/contenidover.asp?contenidoid $=96886$

Brizuela, M., Devoto, S., Ferrucci, G., Garcia, F., Grilli, V., Marco, M., . . . Uranga, M. (11 de 02 de 2021). Sociedad Argentina de Infectologia. Recuperado el 14 de 08 de 2021, de https://www. sadi.org.ar/comisiones-de-trabajo/comision-de-infecciones-en-pediatria/item/1380-sindrome-inflamatorio-multisistemico-asociado-a-infeccion-por-sars-cov-2

Centro para el Control y la Prevención de Enfermedades (CDC). (13 de 11 de 2020). Centro para el Control y la Prevención de Enfermedades (CDC). Recuperado el 25 de 08 de 2021, de https://espanol.cdc.gov/mis/mis-a.html

Clinica Mayo. (24 de 03 de 2021). Clinica Mayo. Recuperado el 12 de 08 de 2021, de https://www. mayoclinic.org/es-es/diseases-conditions/mis-cin-kids-covid-19/symptoms-causes/syc-20502550

Davogustto, G., Clark, D., \& Hardison, E. (19 de 05 de 2021). SÍNDROME INFLAMATORIO MULTISISTÉMICO EN ADULTOS POSCOVID-19. JAMA Network Open, 4(5). doi:doi: 10.1001/jamanetworkopen.2021.10323

Elouardi , Y., Rebahi, H., Zarrouki, A., Ziadi, S., Younous, M., \& Samkaoui, A. (29 de 12 de 2020). Síndrome inflamatorio multisistémico de tipo Kawasaki. Revista Española de Anestesiología y Reanimación. doi:https://doi.org/10.1016/j.redar.2020.11.013 
Fox, M. (03 de 10 de 2020). CNN EN ESPAÑOL. Recuperado el 14 de 08 de 2021, de https://cnnespanol.cnn.com/2020/10/03/los-cdc-identifican-un-nuevo-sindrome-del-covid-19-en-adultossimilar-al-mis-c-en-ninos/

García, M. (21 de 06 de 2021). Redacción Médica. Recuperado el 12 de 08 de 2021, de https://www. redaccionmedica.com/secciones/sanidad-hoy/ el-sindrome-inflamatorio-multisistemico-postcovid-es-posible-en-adultos-1638

Giraldo, C., Tamayo, C., López , E., Caicedo, M., \& Piñeres, B. (21 de 11 de 2020). Síndrome inflamatorio multisistémico en niños asociado a COVID-19. Revisión narrativa de la literatura a propósito de un caso. Acta Colombiana de Cuidado Intensivo. doi:10.1016/j.acci.2020.11.002

Heredia, R. (25 de 05 de 2021). Infomed. Recuperado el 11 de 08 de 2021, de https://infomed.com. ar/caracteristicas-asociadas-al-sindrome-inflamatorio-multisistemico-entre-los-adultos-con-infeccion-por-sars-cov-2/

Mendoza, J., Zambrano, M., Gomez, M., Bravo, R., Cedeño, J., \& Marchan, M. (02 de 06 de 2021). Síndrome Inflamatorio sistémico asociado a SarsCov2. . Revista Cientifica Mundo de la Investigacion y el Conocimiento (RECIMUNDO), 5(2), 238-247. doi:10.26820/recimundo/5.(2).abril.2021.238-247
Organizacion Mundial de la Salud (OMS). (30 de 04 de 2021). Organizacion Mundial de la Salud (OMS). Recuperado el 11 de 08 de 2021, de https://www.who.int/es/news-room/q-a-detail/coronavirus-disease-covid-19-how-is-it-transmitted

Rivera, S. (16 de 10 de 2020). CDC: Adultos están presentando rara enfermedad inflamatoria de órganos vitales derivada del COVID-19. Los Angeles Times. Recuperado el 14 de 08 de 2021, de https://www.latimes.com/espanol/california/ articulo/2020-10-16/cdc-adultos-estan-presentando-rara-enfermedad-inflamatoria-de-organos-vitales-derivada-del-covid-19

Storch, P., \& Vinagre, S. (19 de 12 de 2020). En Familia. Recuperado el 15 de 08 de 2021, de https:// enfamilia.aeped.es/temas-salud/sindrome-inflamatorio-multisistemico-pediatrico-vinculado-sars\#

Thacker, D. (12 de 05 de 2021). Connecticut Childrens. Recuperado el 14 de 08 de 2021, de https://www.connecticutchildrens.org/health-library/ es/parents/pmis-esp/

Theimer, S. (10 de 11 de 2020). Clinica Nayo. Recuperado el 13 de 08 de 2021, de https://newsnetwork. mayoclinic.org/discussion/descubierto-en-adultos-el-sindrome-inflamatorio-de-los-ninos-relacionado-con-la-covid-19/

\section{CITAR ESTE ARTICULO:}

Mera Cáceres, M. F., Arroyo Valencia, V. T., Rojas Carvajal, C. F., \& Narváez Álvarez, C. M. (2021). Síndrome inflamatorio multisistémico en el adulto. RECIAMUC, 5(3), 132-139. https://doi.org/10.47464/reciamuc/5.(3).agosto.2021.132-139

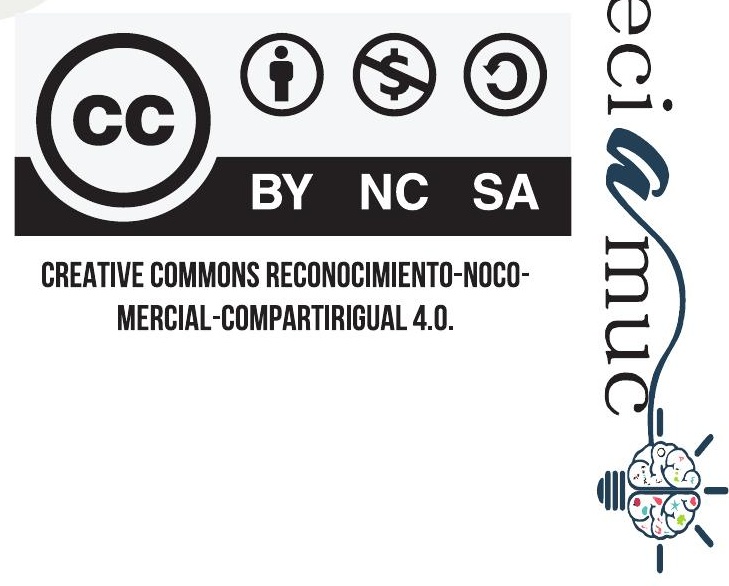

